\title{
Maxwell-modified metric affine gravity
}

\author{
Oktay Cebecioğlu ${ }^{\mathrm{a}}$, Salih Kibaroğlu ${ }^{\mathrm{b}}$ \\ Department of Physics, Kocaeli University, 41380 Kocaeli, Turkey
}

Received: 26 April 2021 / Accepted: 24 September 2021 / Published online: 12 October 2021

(C) The Author(s) 2021

\begin{abstract}
We present a gauge formulation of the special affine algebra extended to include an antisymmetric tensorial generator belonging to the tensor representation of the special linear group. We then obtain a Maxwell modified metric affine gravity action with a cosmological constant term. We find the field equations of the theory and show that the theory reduces to an Einstein-like equation for metric affine gravity with the source added to the gravity equations with cosmological constant $\mu$ contains linear contributions from the new gauge fields. The reduction of the Maxwell metric affine gravity to Riemann-Cartan one is discussed and the shear curvature tensor corresponding to the symmetric part of the special linear connection is identified with the dark energy. Furthermore, the new gauge fields are interpreted as geometrical inflaton vector fields which drive accelerated expansion.
\end{abstract}

\section{Introduction}

It is verified by the Solar System and cosmological tests that general relativity provides an elegant and powerful formulation of gravitation in terms of Riemannian geometry and forms our understanding of space-time [1]. Despite these successes, there are some reasons to believe that general relativity is unable to explain some gravity phenomena on both atomic and cosmological scales and should be either modified or replaced by a new theory of gravity. Recently many papers propose new types of dynamics to explain the dark energy phenomenon [2,3] as well as the dynamical role of the cosmological constant $[4,5]$.

It is also known that the cosmological term, usually associated with the vacuum energy density, cannot be a valid theoretical explanation for the accelerated expansion of the universe [6]. A very different approach holds that cosmic

\footnotetext{
${ }^{a}$ e-mail: ocebecioglu@kocaeli.edu.tr (corresponding author)

b e-mail: salihkibaroglu@gmail.com
}

acceleration is a manifestation of new gravitational physics rather than dark energy, i.e., that it involves a modification of the geometry as opposed to the stress-energy tensor side of the Einstein equations [4]. It is important to point out that one can accommodate a generalized cosmological constant in the gravity theory using extended algebras. A way of introducing the generalized cosmological constant term using the Maxwell algebra was presented in [7] and even more, interestingly it has been argued that by making use of the gauged Maxwell algebra one can understand it as a source of an additional contribution to the cosmological term in Einstein gravity.

Maxwell symmetry was introduced around forty years ago $[8,9]$, but it is only recently that has attracted more attention after the work of Soroka [10] in 2005. The Maxwell symmetry is the result of extending the Poincare symmetry by six additional tensorial Abelian symmetry generators that make the four-momenta non-commutative. Since then a variety of different Maxwell (super) symmetry algebras with interesting geometric and physical properties have been constructed and analyzed in the papers [11-19].

By gauging Maxwell symmetries, one can define modified gravitational theories that extend general relativity by including a generalized cosmological term [20-30]. Among these is the Maxwell extension of special affine symmetry and its gauging which will be the focus of our attention in this paper.

In 1974 Yang [31] put forward a gauge theory of gravity based on the affine group to construct a theory of (quantum) gravity in the high energy limit [32]. On the other hand, in nature, there is no conservation law corresponding to the (special) linear transformation and so the linear transformations must be dynamical, i.e., spontaneously broken [33]. Correspondingly, the papers [34-38] suggested that the renormalizability and unitarity problems in quantum gravity can be overcome by taking the affine group as the dynamical group in a gauge theory of gravity with the help of generalized linear connection [39]. There exists a series of papers 
$[38,40-46]$ in which an affine gauge gravitation theory is considered.

Our paper has the following structure. In Sect. 2, following [28,29], we briefly review the Maxwell extension of the special-affine group, $\mathcal{M S} \mathcal{A}(4, R)$. We also present the transformation rules for the generalized coordinates (coset parameters) and the corresponding differential realization of generators using the nonlinear realization technique. In Sect. 3, we gauge the Maxwell special linear algebra $\mathfrak{m s a}(4, R)$ and find the gauge covariant quantities to construct the gaugeinvariant action. In Sect. 4 , we introduced $\mathcal{S} \mathcal{L}(4, R)$ gauge covariant metric tensor in the affine space needed for the metric affine gravity (MAG). In Sect. 5, we propose an action for Maxwell metric affine gravity by using Euler or GaussBonnet type topological action and derive the equations of motion of corresponding action. We present our conclusions in Sect. 6.

\section{Introducing the special-affine algebra and its maxwell extension}

We begin in this section by giving an overview of the Maxwell extension of the special affine group. For a more complete description of the details, the reader is referred to earlier works $[28,29]$. The special affine symmetry group $\mathcal{S} \mathcal{A}(4, R)$ is given by the semi-direct product of the special linear group $\mathcal{S} \mathcal{L}(4, R)$ and the translation group $\mathcal{T}(4)$ and are generated by the fifteen special linear generators $\stackrel{\circ}{L}_{b}{ }_{b}$ and by the four affine translation generators $P_{a}$, respectively. The commutators of the generators obey the following algebra,

$$
\begin{aligned}
{\left[\stackrel{\circ}{L}_{b}^{a}, \stackrel{\circ}{L}_{d}^{c}\right] } & =i\left(\delta_{b}^{c} \stackrel{\circ}{L}_{d}^{a}-\delta_{d}^{a} \stackrel{\circ}{L}_{b}^{c}\right), \\
{\left[\stackrel{\circ}{L}_{b}^{a}, P_{c}\right] } & =-i\left(\delta_{c}^{a} P_{b}-\frac{1}{4} \delta_{b}^{a} P_{c}\right), \\
{\left[P_{a}, P_{b}\right] } & =0 .
\end{aligned}
$$

From this algebra, we can construct a group element by exponentiation,

$g(x, \stackrel{\circ}{\omega})=e^{i x^{a}(x) P_{a}} e^{i \stackrel{\circ}{b}_{a}^{b}(x) \stackrel{\circ}{L}_{b}^{a}}$,

where $x^{a}(x), \stackrel{\circ}{\omega}_{a}^{b}(x)$ are the real parameters. The MaurerCartan (MC) 1 -forms is defined as $\Omega=-i g^{-1} d g$, here $g$ is the general element of the $\mathcal{S} \mathcal{A}(4, R)$ group and the structure equation is given by

$d \Omega+\frac{i}{2}[\Omega, \Omega]=0$.

Thus, one can show that the MC 1-forms satisfy following equations,

$0=d \Omega_{P}^{a}+\Omega_{i b}^{a} \wedge \Omega_{P}^{b}-\frac{1}{4} \Omega_{\stackrel{\circ}{L}} \wedge \Omega_{P}^{a}$,

$0=d \Omega_{\stackrel{L}{ }{ }^{\circ}}^{a}+\Omega_{\stackrel{\circ}{L} c}^{a} \wedge \Omega_{\stackrel{L}{L} b}^{c}$, where the MC 1-forms $\Omega^{a}{ }_{P}$ and $\Omega^{a}{ }_{L b}$ correspond to translations and special-linear transformations in affine space-time. By using the MC structure equations (4) and making use of the methods presented in $[12,15]$, one can consider a Maxwell extension of the special affine algebra by the antisymmetric generator $Z_{a b}$. The non-vanishing commutation relations are

$$
\begin{aligned}
{\left[\stackrel{\circ}{L}_{b}^{a}, \stackrel{\circ}{L}_{d}{ }_{d}\right] } & =i\left(\delta_{b}^{c} \stackrel{\circ}{L}_{d}^{a}-\delta_{d}^{a} \stackrel{\circ}{L}_{b}{ }_{b}\right), \\
{\left[\stackrel{\circ}{L}_{b}^{a}, P_{c}\right] } & =-i\left(\delta^{a}{ }_{c} P_{b}-\frac{1}{4} \delta_{b}^{a} P_{c}\right),
\end{aligned}
$$

as well as the Maxwell extension

$$
\begin{aligned}
{\left[P_{a}, P_{b}\right] } & =i Z_{a b}, \\
{\left[\stackrel{\circ}{L}_{b}^{a}, Z_{c d}\right] } & =i\left(\delta^{a}{ }_{d} Z_{b c}-\delta^{a}{ }_{c} Z_{b d}+\frac{1}{2} \delta_{b}^{a} Z_{c d}\right) .
\end{aligned}
$$

The action of space-time symmetries on the fields, obtained as an induced representation, is related to the nonlinear realization of symmetries and are developed in reference [4750]. Therefore, when talking about these systems we find the coset construction provides the appropriate language. We refer the reader to references $[33,38,42,46]$ for the derivation of the non-linear realisation with the $\mathcal{S} \mathcal{A}(4, R)$ group. We now construct the non-linear realization corresponding to the group $\mathcal{M S} \mathcal{A}(4, R)$ taking the $\mathcal{S} \mathcal{L}(4, R)$ to be a local symmetry. We therefore parametrize the coset elements of the form

$K(x, \theta)=\frac{\mathcal{M S A}}{S L}=e^{i x \cdot P} e^{i \theta \cdot Z}$,

where $x^{a}, \theta^{a b}$ are the coset parameters. Upon using the definition of the transformation of the coset representative

$g(a, \varepsilon, u) K(x, \theta)=K\left(x^{\prime}, \theta^{\prime}\right) h(\stackrel{\circ}{\omega}$,

where $h(\stackrel{\circ}{\omega})=e^{i \stackrel{\circ}{\omega}_{a}^{b} \stackrel{\circ}{L}_{b}^{a}}$ stands for the subgroup element, we find that the infinitesimal transformations of the coset parameters are given by

$$
\begin{aligned}
\delta x^{a} & =a^{a}+u_{c}^{a} x^{c}-\frac{1}{4} u x^{a}, \\
\delta \theta^{a b} & =\varepsilon^{a b}+u_{c}^{[a \mid} \theta^{c \mid b]}-\frac{1}{2} u \theta^{a b}-\frac{1}{4} a^{[a} x^{b]}, \\
\stackrel{\circ}{a}_{b}{ }_{b} & =u_{b}^{a},
\end{aligned}
$$

where the square brackets denote antisymmetrization of corresponding indices and $a, \epsilon, u$ are the real parameters for affine space-time translations, tensorial translations and special linear symmetry transformation respectively.

For the sake of completeness, we give the differential realization of the symmetry generators

$$
P_{a}=i\left(\partial_{a}-\frac{1}{2} x^{b} \partial_{a b}\right),
$$


$Z_{a b}=i \partial_{a b}$,

$\stackrel{\circ}{L}_{b}^{a}=i\left(x^{a} \partial_{b}+2 \theta^{a c} \partial_{b c}\right)-\frac{1}{4} \delta_{b}^{a}\left(x^{c} \partial_{c}+2 \theta^{c d} \partial_{c d}\right)$,

where $\theta$ derivative is defined by $\partial_{a b} \theta^{c d}=\frac{1}{2}\left(\delta^{c}{ }_{a} \delta_{b}^{d}-\delta_{b}^{c} \delta_{a}^{d}\right)$. It is an easy task to check that the generators satisfy the algebra Eqs. (5) and (6).

\section{Gauging the Maxwell-special-affine algebra}

Let us construct a gauge theory for the Maxwell special affine algebra $\mathfrak{m s a}(4, R)$. For this purpose, we follow the same methods given in $[18,22,28]$. The gauge field is a $\mathfrak{m s a}(4, R)$ algebra valued one-form

$\mathcal{A}=e^{a} P_{a}+B^{a b} Z_{a b}+\stackrel{\circ}{\omega}_{a}^{b} \stackrel{\circ}{L}_{b}^{a}$.

An infinitesimal gauge parameter is

$\zeta(x)=y^{a}(x) P_{a}+\varphi^{a b}(x) Z_{a b}+\lambda_{a}^{b}(x) \dot{L}_{b}^{a}$,

where $y^{a}(x), \varphi^{a b}(x)$, and $\lambda_{a}^{b}(x)$ are the infinitesimal parameters corresponding to the affine translation, tensorial and special linear transformations respectively.

The gauge transformation are given by

$\delta \mathcal{A}=-d \zeta-i[\mathcal{A}, \zeta]$,

evaluating (13), we get

$$
\begin{aligned}
& \delta e^{a}=-d y^{a}-\stackrel{\circ}{\omega}_{b}^{a} y^{b}
\end{aligned}
$$

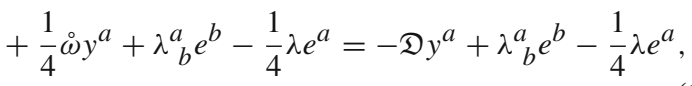

$$
\begin{aligned}
& \delta B^{a b}=-d \varphi^{a b} \\
& -\stackrel{\circ}{\omega}_{c}^{[a \mid} \varphi^{c b]}+\frac{1}{2} \stackrel{\omega}{a b}+\lambda_{c}^{[a \mid} B^{c b]}-\frac{1}{2} \lambda B^{a b}+\frac{1}{2} e^{[a} y^{b]} \\
& =-\mathfrak{D} \varphi^{a b}+\lambda_{c}^{[a \mid} B^{c b]}-\frac{1}{2} \lambda B^{a b}+\frac{1}{2} e^{[a} y^{b]}, \\
& \delta \dot{\omega}^{a}{ }_{b}=-d \lambda^{a}{ }_{b} \\
& -\check{\omega}^{a}{ }_{c} \lambda_{b}^{c}+\stackrel{\circ}{\omega}_{b}^{c} \lambda^{a}{ }_{c}=-\mathfrak{D} \lambda_{b}^{a},
\end{aligned}
$$

where the $\mathcal{S} \mathcal{L}(4, R)$ valued exterior covariant derivative $\mathfrak{D}$ of a tensor density $\Phi$ of affine weight $w$ contains $\left\{w \operatorname{Tr}\left(\omega_{b}^{a}\right) \Phi\right\}$, e.g.

$$
(\mathfrak{D} \Phi)_{b}^{a}=\left[\delta_{b}^{a} d+\stackrel{\circ}{\omega}_{b}^{a}+w(\Phi) \operatorname{Tr}\left(\stackrel{\odot}{b}_{b}^{a}\right)\right] \Phi .
$$

From transformation rules, we immediately infer that 1forms $e^{a}, B^{a b}$, and $\stackrel{\circ}{\omega}_{a}^{b}$ have the following affine scaling weights $-1 / 4,-1 / 2$, and 0 respectively.

Now, acting the exterior covariant derivative on $\mathcal{A}$ we obtain the curvature $\digamma$ satisfying the structure equation and the Bianchi identity

$\digamma=d \mathcal{A}+\frac{i}{2}[\mathcal{A}, \mathcal{A}]$,

$d \digamma+i[\mathcal{A}, \digamma]=0$,

where $d$ is the exterior differential. Upon expressing the curvature form $\digamma$ as

$\digamma=\mathcal{F}^{a} P_{a}+\mathcal{F}^{a b} Z_{a b}+\mathcal{R}_{b}^{a} \stackrel{\circ}{L}_{a}^{b}$,

the structure Eq. (21) becomes

$$
\begin{aligned}
& \mathcal{F}^{a}=d e^{a}+\stackrel{\circ}{\omega}_{b}^{a} \wedge e^{b}-\frac{1}{4} \stackrel{\varrho}{\wedge} e^{a}=\mathfrak{D} e^{a}, \\
& \mathcal{F}^{a b}=d B^{a b}+\stackrel{\circ}{\omega}_{c}^{[a \mid} \wedge B^{c \mid b]}-\frac{1}{2} \stackrel{\circ}{ } \wedge B^{a b}-\frac{1}{2} e^{a} \wedge e^{b}, \\
& =\mathfrak{D} B^{a b}-\frac{1}{2} e^{a} \wedge e^{b}, \\
& \mathcal{R}_{b}^{a}=d \stackrel{\circ}{\omega}_{b}^{a}+\stackrel{\circ}{\omega}_{c}^{a} \wedge \stackrel{\omega}{c}_{b}^{c}=\mathfrak{D} \stackrel{\circ}{ }_{b}^{a} .
\end{aligned}
$$

Thus the curvature forms corresponding to the various generators of the algebra are $\left(\mathcal{F}^{a}, \mathcal{F}^{a b}, \mathcal{R}_{b}^{a}\right)$, and they represent the torsion, the field strength associated with the $B^{a b}$ field and the non-Riemannian affine curvature form, respectively. One concludes that the affine curvature $\mathcal{R}_{b}^{a}$ and the torsion $\mathcal{F}^{a}$ are given by the exterior covariant derivatives of the affine connection and vierbein respectively. On the other hand, the curvature 2-form $\mathcal{F}^{a b}$ coming from Maxwell extension is not given by the exterior covariant derivative of the corresponding gauge field. The extra term in $\mathcal{F}^{a b}$ represents the curvature of the local tensor space. This contribution is present because the commutator of two infinitesimal affine translations equals to an element of the tensor space. Moreover, from the Bianchi identity Eq. (22), we get the following equations

$$
\begin{aligned}
\mathfrak{D F}^{a b} & =\mathcal{R}^{[a \mid} \wedge B^{c \mid b]}-\frac{1}{2} \mathcal{R} \wedge B^{a b}-\frac{1}{2} \mathcal{F}^{[a} \wedge e^{b]}, \\
\mathfrak{D F} \mathcal{F}^{a} & =\mathcal{R}^{a}{ }_{b} \wedge e^{b}-\frac{1}{4} \mathcal{R} \wedge e^{a}, \\
\mathfrak{D R}^{a}{ }_{b} & =0 .
\end{aligned}
$$

Under infinitesimal gauge transformations with parameter $\zeta$, the curvature 2-form $\digamma$ transform as

$\delta \digamma=i[\zeta, \digamma]$,

and hence one gets

$$
\begin{aligned}
\delta \mathcal{F}^{a} & =-\mathcal{R}_{b}^{a}{ }_{b} y^{b}+\frac{1}{4} \mathcal{R} y^{a}+\lambda_{b}^{a} \mathcal{F}^{b}-\frac{1}{4} \lambda \mathcal{F}^{a} \\
\delta \mathcal{F}^{a b} & =-\mathcal{R}_{c}^{[a \mid} \varphi^{c \mid b]}+\frac{1}{2} \mathcal{R} \varphi^{a b}+\lambda_{c}^{[a \mid} \mathcal{F}^{c \mid b]}-\frac{1}{2} \lambda \mathcal{F}^{a b}+\frac{1}{2} \mathcal{F}^{[a} y^{b]},
\end{aligned}
$$

$\delta \mathcal{R}_{b}^{a}=\lambda^{a}{ }_{c} \mathcal{R}_{b}^{c}-\lambda_{b}^{c} \mathcal{R}^{a}{ }_{c}$.

Again from these transformation rules, one observes that curvature 2 -form $\mathcal{F}^{a}, \mathcal{F}^{a b}$, and $\mathcal{R}_{b}^{a}$ have the following affine 
scaling weights $-1 / 4,-1 / 2$, and 0 respectively and they will be useful for constructing invariant Lagrangian densities.

\section{Construction of the metric for the affine space}

Using the definition of the local metric,

$g^{a b}(x)=e^{a} \otimes e^{b}$,

one deduces $\mathcal{S} \mathcal{L}(4, R)$ gauge variation of the metric tensor with the help of Eq. (16) by omitting diffeomorphism part

$$
\begin{aligned}
\delta_{\lambda} g^{a b} & =\left(\lambda_{c}^{a} e^{c}-\frac{1}{4} \lambda e^{a}\right) \otimes e^{b}+e^{a} \otimes\left(\lambda_{c}^{b} e^{c}-\frac{1}{4} \lambda e^{b}\right) \\
& =\lambda^{(a}{ }_{c} g^{c b)}-\frac{1}{2} \lambda g^{a b},
\end{aligned}
$$

where round brackets denote symmetrization. Similarly from the definition of the Kronecker delta tensor

$\delta_{b}^{a}=e^{a} \otimes e_{b}$,

one can obtain the $\mathcal{S} \mathcal{L}(4, R)$ gauge variation of $e_{a}$ as

$\delta_{\lambda} e_{a}=-\lambda^{b}{ }_{a} e_{b}+\frac{1}{4} \lambda e_{a}$,

and the last equation implies

$\delta_{\lambda} g_{a b}=-\lambda_{(a}^{c} g_{c b)}+\frac{1}{2} \lambda g_{a b}$.

With the use of vierbein and local metric, the $\mathcal{S} \mathcal{L}(4, R)$ gauge variation of the coordinate metric becomes

$$
\begin{aligned}
\delta_{\lambda} g_{\mu \nu}(x)= & \left(-\lambda_{(a}^{c} g_{c b)}+\frac{1}{2} \lambda g_{a b}\right) e_{\mu}^{a} e_{\nu}^{b}+g_{a b}\left(\lambda_{c}^{a} e_{\mu}^{c}-\frac{1}{4} \lambda e_{\mu}^{a}\right) e_{\nu}^{b} \\
& +g_{a b} e_{\mu}^{a}\left(\lambda_{c}^{b} e_{\nu}^{c}-\frac{1}{4} \lambda e_{\nu}^{b}\right)=0 .
\end{aligned}
$$

Moreover, the gauge variation of determinant of the vierbein is

$\delta_{\lambda} e=\frac{1}{2} e g^{\mu \nu} \delta_{\lambda} g_{\mu \nu}=0$.

Defining the fully antisymmetric tensor $\eta_{a b c d}$ by

$\eta_{a b c d}=e \varepsilon_{a b c d}$,

where $\varepsilon_{a b c d}$ is the Levi-Civita symbol, its variation under local $\mathcal{S} \mathcal{L}(4, R)$ transformation becomes

$$
\begin{gathered}
\delta_{\lambda} \eta_{a b c d}=-\lambda_{a}^{e} \eta_{e b c d}-\lambda_{b}^{e} \eta_{a e c d}-\lambda_{c}^{e} \eta_{a b e d} \\
-\lambda_{d}^{e} \eta_{a b c e}+\lambda \eta_{a b c d}=0,
\end{gathered}
$$

and has affine scaling weight 1 .

Having defined the local metric for the affine space-time, the metricity is obtained by taking the covariant derivative of the local metric, i.e., $Q^{a b}=\mathcal{D} g^{a b}$ and its explicit form follows

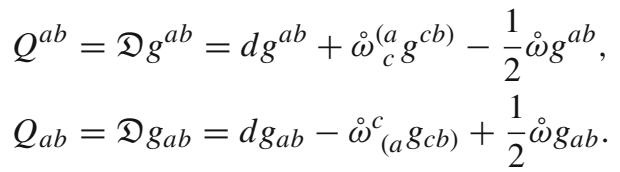

This in turn leads to the covariant derivative of the metricity

$\mathfrak{D} Q^{a b}=\mathcal{R}^{(a}{ }_{c}{ }^{c b)}-\frac{1}{2} \mathcal{R} g^{a b}$.

Likewise,

$\mathfrak{D} Q_{a b}=-\mathcal{R}_{(a}^{c}{ }_{(a b)}+\frac{1}{2} \mathcal{R} g_{a b}$.

\section{Maxwell-modified mag field equations}

One way of constructing the action is to begin from the covariant quantities with manifest geometric meanings. To prescribe the dynamics of the gauge fields, we have to introduce an action, invariant under local $\mathcal{S} \mathcal{L}(4, R)$ transformation. We need then curvatures $\mathcal{R}_{b}^{a}, \mathcal{F}^{a b}$ and the metric $g^{a b}$ obtained in the last section. We start with following topological action,

$$
S=\frac{1}{2 \chi} \int \mathcal{J} \wedge * \mathcal{J}=\frac{1}{4 \chi} \int \eta_{a b c d} \mathcal{J}^{a b} \wedge \mathcal{J}^{c d},
$$

known as Euler or Gauss-Bonnet type action, where $\chi=$ $8 \pi G / c^{4}$ is the Einstein's constant, $(*)$ is the Hodge dual and $\eta_{a b c d}$ is defined by Eq. (40). Contracting $\mathcal{R}_{b}^{a}$ with $g^{a b}$, we can form curvature 2-form $\mathcal{R}^{a b}=\mathcal{R}_{c}^{a} g^{c b}$ and it's gauge transformation is given by

$\delta \mathcal{R}^{a b}=\lambda^{a} \mathcal{R}^{c b}+\lambda^{b}{ }_{c} \mathcal{R}^{a c}-\frac{1}{2} \lambda \mathcal{R}^{a b}$.

It has the same form as Eq. (31) when the diffeomorphism part omitted. So, one can introduce a shifted curvature 2form,

$\mathcal{J}^{a b}=\mathcal{R}^{a b}-\mu \mathcal{F}^{a b}$,

where $\mu$ is a dimensionful constant. Its gauge transformation becomes

$\delta \mathcal{J}^{a b}=\lambda_{c}^{a} \mathcal{J}^{c b}+\lambda^{b}{ }_{c} \mathcal{J}^{a c}-\frac{1}{2} \lambda \mathcal{J}^{a b}$.

The gauge transformation of $* \mathcal{J}_{a b}=\frac{1}{2} \eta_{a b c d} \mathcal{J}^{c d}$ has the following form

$\delta * \mathcal{J}_{a b}=\frac{1}{2}\left(\lambda^{e}{ }_{c} \eta_{a b e d}+\lambda_{d}^{e} \eta_{a b c e}-\frac{1}{2} \lambda \eta_{a b c d}\right) \mathcal{J}^{c d}$,

the term in the parentheses can be written another form after re-indexing the labels as 


$$
\begin{aligned}
\lambda_{c}^{e} \eta_{\text {abed }}+\lambda^{e}{ }_{d} \eta_{a b c e}-\frac{1}{2} \lambda \eta_{a b c d}= & -\lambda_{a}^{e} \eta_{e b c d}-\lambda^{e}{ }_{b} \eta_{a e c d} \\
& +\frac{1}{2} \lambda \eta_{a b c d},
\end{aligned}
$$

then variation of the Hodge dual of $J$ becomes

$\delta * \mathcal{J}=-\lambda^{e}{ }_{a} * \mathcal{J}_{e b}-\lambda_{b}^{e} * \mathcal{J}_{a e}+\frac{1}{2} \lambda * \mathcal{J}_{a b}$.

Invariance of the action under gauge transformation can be checked easily with the help of Eqs. (47) and (50). By construction, the action is automatically invariant under diffeomorphism and has affine scaling weight zero. Before introducing dynamics, we still have to discuss the nature of the gauge and general coordinate transformations (diffeomorphism). From a gauge theory perspective, infinitesimal displacements are a "local translation". Under a local translation, quantities change according to Lie derivative. Indeed using the identity $l_{y} \sigma=i_{y} d \sigma+d i_{y} \sigma$ for the Lie derivative of any one-form $\sigma$, we easily find that

$$
\begin{aligned}
\delta_{\text {diff }} e^{a}= & i_{y} \mathcal{F}^{a}-\mathfrak{D} y^{a}+\lambda^{a}{ }_{b} e^{b}-\frac{1}{4} \lambda e^{a}=i_{y} \mathcal{F}^{a}+\delta e^{a} \\
\delta_{\text {diff } B^{a b}=} & i_{y} \mathcal{F}^{a b}-\mathfrak{D} \varphi^{a b} \\
& +\lambda^{[a \mid}{ }_{c} \mathcal{F}^{c \mid b]}-\frac{1}{2} \lambda B^{a b}-\frac{1}{2} y^{[a} e^{b]}=i_{y} \mathcal{F}^{a b}+\delta B^{a b}
\end{aligned}
$$

$\delta_{\operatorname{diff}} \stackrel{\circ}{\omega}_{a}^{b}=i_{y} \mathcal{R}_{b}^{a}-\mathfrak{D} \lambda_{b}^{a}=i_{y} \mathcal{R}_{b}^{a}+\delta \stackrel{\circ}{b}_{a}$

In particular if the affine torsion has vanishing projection along the tangent vector $y$, then the diffeomorphism coincides with gauge transformation. Torsion constraint also allows one to replace local translations by general coordinate transformations. Invariance of the action (44) under local translation can be directly checked by using the explicit form of the Lie derivative:

$\delta_{\text {diff }} S=\int l_{y} L=\int d i_{y} L+i_{y} d L$

The Lagrangian is a 4-form density, so the first term is a total divergence can be ignored as a surface term and the second term being a 5-form vanishes identically on the 4D space-time.

It remains, of course, to find the field equations for the gauge fields. The variation of the action (44) with respect to gauge fields $\stackrel{\circ}{\omega}_{a}^{b}(x), e^{a}(x), B^{a b}(x)$ and the metric $g^{a b}(x)$ lead to the following equations:

$$
\begin{aligned}
& \mathfrak{D}\left(g^{a c} * \mathcal{J}_{b c}\right)-2 \mu B^{a c} \wedge * \mathcal{J}_{b c}=0, \\
& e^{b} \wedge * \mathcal{J}_{a b}=0, \\
& \mathfrak{D}\left(* \mathcal{J}_{a b}\right)=0, \\
& \mathcal{R}_{(a}^{c} \wedge * \mathcal{J}_{c b)}-\frac{1}{2} g_{a b} \mathcal{J}^{c d} \wedge * \mathcal{J}_{c d}=0 .
\end{aligned}
$$

It is important to note that these equations of motion transform as $\mathcal{S} \mathcal{L}(4, R)$-tensors. Here, the first and second equa- tions represent the generalizations of the torsion equation, and the Einstein equation. The third equation arises from the Maxwell symmetry and the last equation is the generalized version of the equation of motion for the metric tensor given in [43].

In order to write Eq. (56) in the more conventional form, one switches from tangent indices to coordinate indices,

$$
\begin{aligned}
* \mathcal{J}_{c d} \wedge e^{c} & =\frac{1}{2} e \varepsilon_{a b c d} \mathcal{J}^{a b} \wedge e^{c} \\
& =\frac{1}{4} e \varepsilon_{a b c d} \mathcal{J}_{\mu \nu}^{a b} e_{\alpha}^{c} d x^{\mu} \wedge d x^{\nu} \wedge d x^{\alpha},
\end{aligned}
$$

multiplying this from right $d x^{\beta}$, we get following equation

$\mathcal{J}_{v}^{\mu}-\frac{1}{2} \delta^{\mu} \mathcal{J}=0$

which can also be expressed as

$\mathcal{R}^{\mu}{ }_{v}-\frac{1}{2} \delta^{\mu}{ }_{v} \mathcal{R}=\mu\left(\mathcal{F}^{\mu}{ }_{v}-\frac{1}{2} \delta^{\mu}{ }_{\nu} \mathcal{F}\right)$

This has resemblance to the usual Einstein's field equation. However, the curvature tensor $\mathcal{R}^{\mu}{ }_{v}$ and $\mathcal{F}^{\mu}{ }_{v}$ may not necessarily be symmetric. $\mathcal{F}^{\mu}{ }_{\nu}$ acts as sources in the field equation of gravity. This equation can be written in a more familiar form by going from differential form to space-time tensors as

$$
\begin{aligned}
& \frac{1}{2} \mathcal{F}_{\rho \sigma}^{\mu \nu} d x^{\rho} \wedge d x^{\sigma} \\
& \quad=\frac{1}{2}\left(e_{a}^{\mu} e_{b}^{v} \mathfrak{D}_{[\rho} B_{\sigma]}^{a b}-\frac{1}{2} \delta_{\rho}^{\mu} \delta_{\sigma}^{\nu}+\frac{1}{2} \delta_{\sigma}^{\mu} \delta_{\rho}^{\nu}\right) d x^{\rho} \wedge d x^{\sigma},
\end{aligned}
$$

so we get explicit form of $\mathcal{F}_{\rho \sigma}^{\mu \nu}$,

$\mathcal{F}_{\rho \sigma}^{\mu \nu}=e_{a}^{\mu} e_{b}^{v} \mathfrak{D}_{[\rho} B_{\sigma]}^{a b}-\frac{1}{2} \delta_{\rho}^{\mu} \delta_{\sigma}^{\nu}+\frac{1}{2} \delta_{\sigma}^{\mu} \delta_{\rho}^{\nu}$,

then $F_{\rho}^{\mu}$ and $F$ can be extracted respectively as,

$\mathcal{F}_{\rho}^{\mu}=\mathcal{F}_{\rho v}^{\mu v}=e_{a}^{\mu} e_{b}^{v} \mathfrak{D}_{[\rho} B_{v]}^{a b}-\frac{3}{2} \delta_{\rho}^{\mu}$,

$\mathcal{F}=\mathcal{F}_{\mu}^{\mu}=e_{a}^{\mu} e_{b}^{v} \mathfrak{D}_{[\mu} B_{\nu]}^{a b}-6$.

Thanks to the last three equations, we can re-expressed the right hand side of Eq. (61),

$\mathcal{F}_{v}^{\mu}-\frac{1}{2} \delta^{\mu}{ }_{v} \mathcal{F}=e_{a}^{\mu} e_{b}^{\rho} \mathfrak{D}_{[v} B_{\rho]}^{a b}-\frac{1}{2} \delta_{v}^{\mu} e_{a}^{\rho} e_{b}^{\sigma} \mathfrak{D}_{[\rho} B_{\sigma]}^{a b}+\frac{3}{2} \delta_{v}^{\mu}$,

so the Eq. (61) takes the following form,

$\mathcal{R}_{\nu}^{\mu}-\frac{1}{2} \delta_{v}^{\mu} \mathcal{R}-\frac{3}{2} \mu \delta_{v}^{\mu}=\mu\left(e_{a}^{\mu} e_{b}^{\rho} \mathfrak{D}_{[v} B_{\rho]}^{a b}-\delta_{v}^{\mu} e_{a}^{\rho} e_{b}^{\sigma} \mathfrak{D}_{\rho} B_{\sigma}^{a b}\right)$,

where $\frac{1}{2} \delta_{\nu}^{\mu} e_{a}^{\rho} e_{b}^{\sigma} \mathfrak{D}_{[\rho} B_{\sigma]}^{a b}=\delta_{\nu}^{\mu} e_{a}^{\rho} e_{b}^{\sigma} \mathfrak{D}_{\rho} B_{\sigma}^{a b}$. 
We see that the source added to the gravity equations with cosmological constant $\mu$ contains linear contributions from the new gauge fields. The second term on the right-hand side of (67) provides a field-dependent modification of the cosmological constant at the left-hand side of the equation[30].

To the decomposition above there corresponds a splitting of the connection 1-form into its Riemannian and nonRiemannian parts $\omega_{b}^{a}$ and $v_{b}^{a}$, respectively, as

$\stackrel{\circ}{\omega}_{a}^{b}=\omega_{b}^{a}+v_{b}^{a}$,

where $\omega_{b}^{a}$ is antisymmetric Lorentz connection and $v_{b}^{a}$ is symmetric shear connection. In terms of these forms Eq. (67) becomes,

$$
\begin{aligned}
& \mathcal{R}_{\nu}^{\mu}-\frac{1}{2} \delta_{\nu}^{\mu} \mathcal{R}-\frac{3}{2} \mu \delta_{\nu}^{\mu} \\
&=\mu e_{a}^{\mu} e_{b}^{\rho}\left(D_{[\nu} B_{\rho]}^{a b}+v_{[v c}^{[a} \wedge B_{\rho]}^{c b]}-\frac{1}{2} v_{[\nu} B_{\rho]}^{a b}\right) \\
&-\mu \delta_{\nu}^{\mu} e_{a}^{\rho} e_{b}^{\sigma}\left(D_{\rho} B_{\sigma}^{a b}+v_{\rho c}^{[a} \wedge B_{\sigma}^{c b]}-\frac{1}{2} v_{\rho} B_{\sigma}^{a b}\right),
\end{aligned}
$$

where $D$ is the Lorentz exterior covariant derivative. We see that this is simply Einstein's equation for metric affine gravity with a cosmological constant term. It is then sensible to identify the expression in the curly bracket as the source of the gravitational field. Note also that, if the affine curvature tensor is decomposed into the Riemannian and shear strength tensor parts, it can end up in a Riemann-Cartan theory with two extra fields, i.e., translational connection and the symmetric sector of the $\mathcal{S} \mathcal{L}(4, R)$-connection both migrate to the stress-energy sector. This means that shear strength tensor, considered to be an intrinsic property of metric affine spacetime, may measure the energy content of the universe, i.e., dark energy.

\section{Conclusion}

In conclusion, we have used coset formalism to determine gauge formulation of the Maxwell extended special affine gravity. For this purpose, we have introduced a $\mathcal{S} \mathcal{L}(4, R)$ connection over a $\left(x^{a}, \theta^{a b}\right)=(4,6)$-dimensional tensor extended space obtained by extending a metric affine spacetime with six tensor coordinates. After gauging the Maxwell special affine group $\mathcal{M S} \mathcal{A}(4, R)$, we propose a locally $\mathcal{S L}(4, R)$ invariant action for the Maxwell extended MAG with the help of topological Euler or Gauss-Bonnet type action.

It is found that the Maxwell extension modifies the results of the metric affine gravity not only by changing the numerical coefficient of the cosmological term but also the new abelian gauge fields $B_{\mu}^{a b}(x)$ terms already present in the latter theory. These additional terms represent the characteristic contributions of the Maxwell symmetry. To our knowledge, these terms have not been extensively analyzed yet but up to now, the additional terms have been interpreted a lot of meanings. For instance, in the gravitational framework, the additional degrees of freedom represent uniform gauge field strengths in (super)space which leads to uniform constant energy density [13]. Also, it is known that such an additional term may be related to dark energy [4,5]. Moreover, the Maxwell symmetry provides a geometric background to define vector inflatons in cosmological models [30] and the additional terms may be interpreted as geometrical inflaton vector fields which drive accelerated expansion. To sum up, these results show the importance and potential of the Maxwell symmetry.

Acknowledgements This work was supported by the Scientific and Technological Research Council of Turkey (TÜBİTAK) Research Project No. 118F364.

Data Availability Statement This manuscript has no associated data or the data will not be deposited. [Authors' comment: There are no external data associated with the manuscript.]

Open Access This article is licensed under a Creative Commons Attribution 4.0 International License, which permits use, sharing, adaptation, distribution and reproduction in any medium or format, as long as you give appropriate credit to the original author(s) and the source, provide a link to the Creative Commons licence, and indicate if changes were made. The images or other third party material in this article are included in the article's Creative Commons licence, unless indicated otherwise in a credit line to the material. If material is not included in the article's Creative Commons licence and your intended use is not permitted by statutory regulation or exceeds the permitted use, you will need to obtain permission directly from the copyright holder. To view a copy of this licence, visit http://creativecomm ons.org/licenses/by/4.0/.

Funded by SCOAP ${ }^{3}$.

\section{References}

1. C.M. Will, Living Rev. Relativ. 17, 4 (2014)

2. E.J. Copeland, M. Sami, S. Tsujikawa, Int. J. Mod. Phys. D 15, $1753(2001)$

3. R.R. Caldwell, E.V. Linder, Phys. Rev. Lett. 95, 141301 (2005)

4. J. Frieman, M. Turner, D. Huterer, Annu. Rev. Astron. Astrophys. 46, 385-432 (2008)

5. T. Padmanabhan, Adv. Sci. Lett. 2, 174 (2009)

6. J. Sola, J. Phys. Conf. Ser. 453, 012015 (2013)

7. J.A. de Azcárraga, K. Kamimura, J. Lukierski, Phys. Rev. D 83, 124036 (2011)

8. H. Bacry, P. Combe, J.L. Richard, Nuovo Cimento 67, 267-299 (1970)

9. R. Schrader, Fortsch. Phys. 20, 701 (1972)

10. D.V. Soroka, V.A. Soroka, Phys. Lett. B 607, 302-305 (2005)

11. J. Gomis, K. Kamimura, J. Lukierski, JHEP 08, 39 (2009)

12. S. Bonanos, J. Gomis, J. Phys. A Math. Theor. 42, 145206 (2009)

13. S. Bonanos, J. Gomis, K. Kamimura, J. Lukierski, Phys. Rev. Lett. 104, 090401 (2010)

14. S. Bonanos, J. Gomis, K. Kamimura, J. Lukierski, J. Math. Phys. 51, $102301(2010)$ 
15. S. Bonanos, J. Gomis, J. Phys. A Math. Theor. 43, 015201 (2010)

16. R. Durka, J. Kowalski-Glikman, M. Szczachor, Mod. Phys. Lett. A 27, 1250023 (2012)

17. J.A. de Azcárraga, J.M. Izquierdo, J. Lukierski, M. Woronowicz, Nucl. Phys. B 869, 303-314 (2013)

18. J.A. de Azcárraga, J.M. Izquierdo, Nucl. Phys. B 885, 34-45 (2014)

19. S. Fedoruk, J. Lukierski, J. High Energy Phys. 02, 128 (2013)

20. D.V. Soroka, V.A. Soroka, Phys. Lett. B 707, 160-162 (2012)

21. R. Durka, J. Kowalski-Glikman, M. Szczachor, Mod. Phys. Lett. A 26, 2689-2696 (2011)

22. O. Cebecioğlu, S. Kibaroğlu, Phys. Rev. D 90, 084053 (2014)

23. P.K. Concha, E.K. Rodríguez, P. Salgado, JHEP 08, 9 (2015)

24. P. Concha, L. Ravera, E. Rodriguez, JHEP 01, 192 (2019)

25. P. Concha, Phys. Lett. B 792, 290-297 (2019)

26. S. Kibaroğlu, O. Cebecioğlu, Eur. Phys. J. C 79, 898 (2019)

27. S. Kibaroğlu, O. Cebecioğlu, Phys. Lett. B 803, 135295 (2020)

28. O. Cebecioğlu, S. Kibaroğlu, Phys. Lett. B 751, 131 (2015)

29. S. Kibaroğlu, M. Şenay, O. Cebecioğlu, Mod. Phys. Lett. A 34, 1950016 (2019)

30. J. A. de Azcárraga, K. Kamimura, J. Lukiersky, Int. J. Mod. Phys. Conf. Ser. 33, 350-356 (2013)

31. C.N. Yang, Phys. Rev. Lett. 33, 445-447 (1974)

32. F.W. Hehl, J.D. McCrea, E.W. Mielke, Y. Ne'eman, Found. Phys. 19, 1075-1100 (1989)

33. A.B. Borisov, V.I. Ogievetskii, Theor. Math. Phys. 21, 1179-1188 (1974)
34. Y. Ne'eman, Dj. Sijacki, Ann. Phys. N. Y. 120, 292 (1979)

35. Y. Ne'eman, Dj. Sijacki, Phys. Lett. B 200, 489 (1988)

36. Y. Ne'eman, Dj. Sijacki, Phys. Rev. D 37, 3267 (1988)

37. C.-Y. Lee, Y. Ne'eman, Phys. Lett. B 242, 599 (1990)

38. A. Lopez-Pinto, A. Tiemblo, R. Tresguerres, Class. Quantum Gravity 12, 1503-1516 (1995)

39. F.W. Hehl, J.D. McCrea, E.W. Mielke, Y. Ne'eman, Phys. Rep. 258, 1-171 (1995)

40. E. Lord, Phys. Lett. A 65, 1-4 (1978)

41. F.W. Hehl, E.A. Lord, Y. Ne'eman, Phys. Rev. D 17, 428-433 (1978)

42. S. Hamamoto, Prog. Theor. Phys. 60, 1910 (1978)

43. B. Julia, S. Silva, Class. Quantum Gravity 15, 2173-2215 (1998)

44. M. Leclerc, Ann. Phys. 321, 708-743 (2006)

45. R.F. Sobreiro, V.J. Vasquez Otoya, J. Geom. Phys. 61, 137-150 (2011)

46. R. Tresguerres, E.W. Mielke, Phys. Rev. D 62, 044004 (2000)

47. S. Coleman, J. Wess, B. Zumino, Phys. Rev. 177, 2239 (1969)

48. C. Callan, S. Coleman, J. Wess, B. Zumino, Phys. Rev. 177, 2247 (1969)

49. A. Salam, J. Strathdee, Phys. Rev. 184, 1750 (1969)

50. A. Salam, J. Strathdee, Phys. Rev. 184, 1760 (1969) 